TecnoLógicas

ISSN-p 0123-7799

ISSN-e 2256-5337

Vol. 23, No. 48, pp. 1-16

Mayo-agosto de 2020

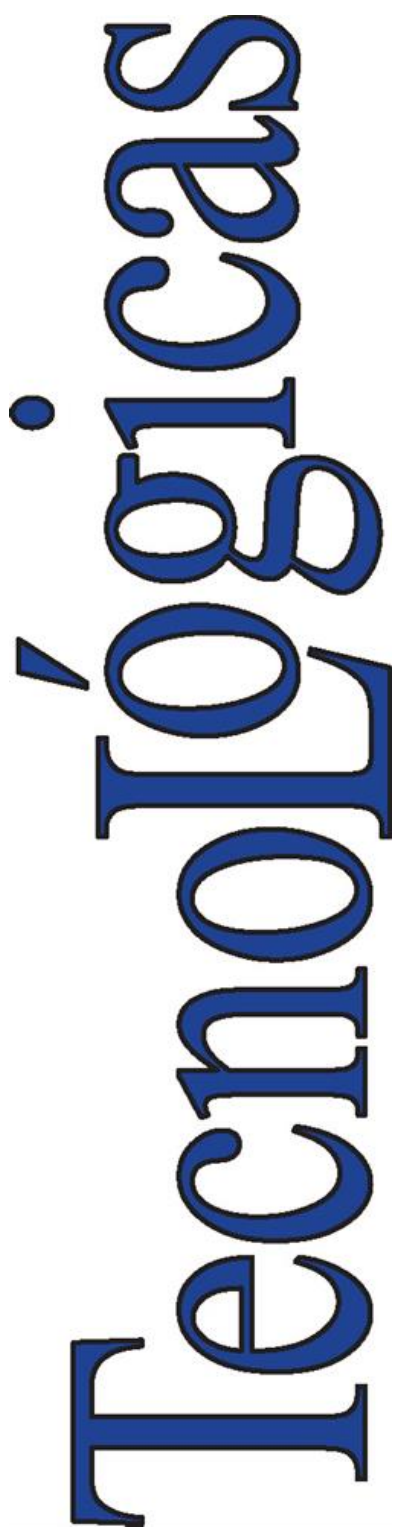

(C) Instituto Tecnológico Metropolitano Este trabajo está licenciado bajo una Licencia Internacional Creative

Commons Atribución (CC BY-NC-SA)

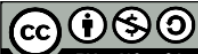

Artículo de Investigación/Research Article

\section{Análisis frecuencial y de la densidad espectral de potencia de la estabilidad de sujetos amputados}

\section{Frequency and Spectral Power Density Analysis of the Stability of Amputees Subjects}

\author{
Lely A. Luengas C. (D) ${ }^{1}$, \\ y Daissy C. Toloza ${ }^{2}$
}

Recibido: 01 de agosto de 2019 Aceptado: 14 de febrero de 2020

Cómo citar / How to cite

L. A. Luengas-C., D. C. Toloza, "Análisis frecuencial y de la densidad espectral de potencia de la estabilidad de sujetos amputados", TecnoLógicas, vol. 23, no. 48, pp. 1-16, 2020.

https://doi.org/10.22430/22565337.1453

1 PhD. en Ingeniería, Facultad Tecnológica, Universidad Distrital Francisco José de Caldas, Bogotá-Colombia, laluengasc@udistrital.edu.co

2 PhD. en Ingeniería, Ingeniería Biomédica, Universidad Manuela Beltrán, Bucaramanga-Colombia, daissy.toloza@umb.edu.co 


\title{
Resumen
}

La amputación transtibial provoca una disminución de la información somatosensorial disponible para el sistema nervioso central, esta pérdida muscular y fisiológica que involucra la articulación del tobillo reduce la fuerza muscular de las piernas, lo que afecta la capacidad de equilibrio y la movilidad de quien la sufre, al presentarse bajo uso de las extremidades inferiores, se provoca una hipotrofia de los músculos y el déficit en su fuerza incrementa. Adicionalmente, los sujetos con amputación transtibial deben adaptarse a la falta de la articulación fisiológica del tobillo y los músculos flexores plantares, partes indispensables para una adecuada movilidad articular, fuerza muscular y capacidad de ajuste activo de la prótesis durante la postura estática de bipedestación. Así, se observa que la reducción de la fuerza muscular está asociada con la disminución del equilibrio. El objetivo de la investigación fue analizar el Centro de Presión (COP) para conocer el comportamiento de la estabilidad en una muestra que consistía de sujetos amputados. Se estudió el análisis armónico de señales de la estabilidad en personas amputadas transtibialmente y usuarios de prótesis, esto con el fin de conocer el comportamiento del centro de presión en dicho conjunto de gente. Dos grupos, cada uno de nueve individuos, fueron analizados: un grupo control de no amputados y un grupo de amputados. Se empleó el periodograma vía método de Welch para hallar las componentes frecuenciales propias del centro de presión bajo cada pie con el fin de caracterizarlas y permitir su detección, y así poder entender las diferencias existentes entre los grupos estudiados. En el presente trabajo se muestra el análisis armónico de la señal no estacionaria, y se argumenta que esta es una ayuda considerable en el análisis de la estabilidad. Los resultados indican que la amputación incide en la Densidad Espectral de Potencia (DEP), ya que existe diferencia entre las frecuencias del lado amputado y el no amputado (mayor oscilación en el lado amputado, eje antero-posterior); igual situación se presenta entre los dos grupos evaluados (mayor potencia en los amputados en todas las condiciones de la prueba).

\section{Palabras clave}

Amputación transtibial, biomecánica, centro de presión, densidad espectral de potencia, estabilidad de sistemas.

\begin{abstract}
Transtibial amputations reduce the amount of somatosensorial information available to the central nervous system. This muscular and physiological loss involving the ankle joint reduces the muscle strength of the legs, which affects the balance and mobility of those who suffer from it. As lower limbs are used less, muscle hypertrophy occurs and the muscle strength deficit increases. Additionally, transtibial amputees should adapt themselves to the lack of a physiological joint between the ankle and the plantar flexor muscles, which are essential for an adequate joint mobility, muscle strength, and active adjustment capacity of the prosthesis during quiet stance. Thus, the reduction of their muscle strength is associated with compromised balance. This study analyzed the Center of Pressure (COP) of a group of transtibial amputees to understand the behavior of their stability. A harmonic analysis of the stability signs of such amputees (prosthesis users) was examined in order to reveal the behavior of their center of pressure. We analyzed two groups of 9 participants each: non-amputee control group and amputees. We used a periodogram, via Welch's method, to find the frequential components of the center of pressure under each foot in order to characterize and detect them and understand the differences between the study groups. This paper presents the harmonic analysis of a nonstationary signal and supports the idea that the latter is an important tool for stability analysis. The results indicate that amputations have an influence on Power Spectral Density (DEP) because there is a difference in frequencies between the amputated and the non-amputated limb (greater oscillation on the amputated side, antero-posterior axis). The same situation occurred between the study groups (greater power in amputees under all testing conditions).
\end{abstract}

\section{Keywords}

Transtibial amputation, biomechanics, center of pressure, power spectral density, system stability. 


\section{INTRODUCCIÓN}

Las amputaciones se dan por diversas causas, como enfermedades, accidentes de toda índole, o por malformaciones durante la gestación; además de esto, cabe aclarar que, en Colombia, una causa común de amputación es debida al conflicto armado que vive el país. Según la Organización Mundial de la Salud (OMS), cerca del 15\% de los habitantes del mundo sufren algún tipo de discapacidad. En Colombia, esta cifra es de $6,3 \%$, siendo que el $10 \%$ de estas personas son amputadas [1].

El Observatorio de Minas de la Vicepresidencia de La República ha registrado más de 11000 víctimas de minas antipersonales (MAP) y municiones sin explotar entre 1990 y 2018, cerca de 2000 de ellos murieron, el resto resultaron heridos, la mayor parte con mutilaciones y amputaciones en sus extremidades [2], [3].

Los amputados por debajo de rodilla o amputados transtibiales utilizan la prótesis transtibial como medio de rehabilitación para su recuperación funcional. La prótesis debe ser adecuada y responder a las necesidades propias de cada persona. El uso de esta requiere de una óptima adaptación protésica, es vital el trabajo realizado por el técnico protesista, pues es quien fija o alinea la prótesis de acuerdo a las necesidades del paciente [4].

La estabilidad es la propiedad de un cuerpo apartado de su equilibrio que trata de volver a su estado inicial, retomando la trayectoria de movimiento anterior a la perturbación. La estabilización postural se caracteriza por oscilaciones corporales reflejadas en la aceleración del centro de masa (COM), esta permite regular la relación entre el COM y la base de soporte y se presenta en la transición hacia la obtención de una postura erecta.

La ubicación de la vertical gravitatoria en la base de sustentación determina el tipo de estabilidad, cuanto más cercana al punto medio de la base de sustentación sea la proyección del centro de gravedad, mayor estabilidad se tiene en el cuerpo.

La evaluación de la estabilidad se realiza con el análisis del centro de presión (COP), pues tal método refleja los ángulos de las articulaciones y por tanto las orientaciones de los segmentos corporales, también las velocidades angulares $y$ aceleraciones conjuntas del cuerpo utilizadas para mantener el centro de gravedad dentro de los límites de la base de soporte. La existencia de grandes desplazamientos del COP está asociada a la inestabilidad postural y la pérdida del equilibrio [5]. El análisis del COP se ha efectuado tanto en el dominio del tiempo como de la frecuencia.

Recientemente se utilizan técnicas encaminadas al análisis no lineal o al estocástico, las cuales permiten obtener otro tipo de información; mientras que los lineales se enfocan en presentar un resumen estadístico de los datos, los nolineales requieren de un análisis y cálculo más complejo para identificar las propiedades estructurales y dinámicas subyacentes de la inestabilidad postural [6]-[8].

Una limitación de movilidad funcional bastante común en las personas con pérdida de extremidades inferiores es un déficit en el control del equilibrio. Las deficiencias en el control del equilibrio se asocian con la reducción en la movilidad funcional de un individuo en el hogar y en la comunidad, como consecuencia, su calidad de vida se ve afectada. Hasta la fecha, las investigaciones sobre el control del equilibrio postural en individuos amputados se realizan, generalmente, con parámetros lineales.

Estos estudios de posturografía han revelado aumentos en las medidas de balanceo postural, incluida la velocidad y el desplazamiento del centro de presión en comparación con personas no amputadas de la misma edad [5]. Sin embargo, las técnicas de análisis lineales no son sensibles a los cambios mínimos de estabilidad presentada por un sujeto, 
tampoco al realizar comparaciones entre grupos de sujetos.

La Densidad Espectral de Potencia (siglas DEP, en inglés Power Spectral Density) de una señal es una función matemática que da a conocer la distribución de la potencia de dicha señal sobre las distintas frecuencias en donde está formada. Así, se puede establecer el rango de frecuencias donde se concentran las variaciones de potencia. La observación del comportamiento de señales en el dominio de la frecuencia resulta de gran ayuda, ya que se pueden discriminar las variaciones más fácilmente que en el dominio del tiempo, esto permite la comparación entre dos grupos poblacionales, y detectar una variación en el comportamiento del parámetro estudiado [9]-[11]. La DEP se ha utilizado en el análisis de eventos clínicos, por ejemplo, en la detección de latidos cardiacos patológicos [12], así como en el análisis del centro de presión en diabéticos con neuropatía periférica. Por ejemplo, el estudio de Oppenheim et al. indicó que la DEP del COP en esa población se encuentra en las frecuencias medias-altas $(0,5-1,0 \mathrm{~Hz})$, producto del deterioro del control postural debido a la deficiente retroalimentación propioceptiva [13], [14] indicó que la frecuencia de potencia media se estableció en $0,33 \mathrm{~Hz}$ para esa misma población.

El propósito de este estudio fue investigar los efectos de la amputación transtibial en la estabilidad estática revelados por el análisis espectral de potencia de la oscilación del COP en posición de bipedestación estática. Se tuvieron en cuenta dos grupos poblacionales, uno corresponde a los amputados transtibiales unilaterales y el otro grupo a los no amputados (grupo control); a partir de esto, se indagó la diferencia en las características del dominio de la frecuencia del COP entre los grupos.

\section{MÉTODOS}

\subsection{Sujetos}

Para analizar la estabilidad en amputados transtibiales, se formaron dos grupos, cada uno de nueve sujetos adultos: uno de control, con no amputados, y uno de estudio, con amputados. Los participantes dieron su consentimiento informado antes de su inclusión en el estudio, este fue avalado por el Comité de Bioética de la Universidad Distrital Francisco José de Caldas, de Bogotá, Colombia.

El grupo de no amputados estuvo compuesto por personas de una edad promedio de 44,32 años (con desviación estándar [SD]: 12,7, rango 25-61 años).

Todos los sujetos son físicamente activos y no reportaron ningún trastorno musculoesquelético o neurológico.

El promedio de la masa corporal fue de 69,44 kg (SD: 14,99, rango 54-94 kg), talla promedio $167,44 \mathrm{~cm}$ (SD: 8,45 , rango 157 $183 \mathrm{~cm}$ ). Se excluyeron los pacientes que presentaban uno o más de los siguientes criterios: haber sido diagnosticado con una enfermedad que afecte el equilibrio, usar medicamentos que perturben el equilibrio, usar ayudas técnicas para mantenerse de pie o para desplazarse, y presentar vértigo o mareo. El grupo de amputados estuvo conformado por amputados transtibiales unilaterales, con amputación por trauma debido a mina antipersonal, todos hombres, con uso adecuado de prótesis por más de un año, con criterios de funcionalidad, sin alteraciones musculoesqueléticas, neurológicas, sensoriales, cognitivas, articulares ni lesiones en la piel.

Los criterios de exclusión para este grupo fueron: uso de algún dispositivo de asistencia para mantenerse de pie o para caminar, presencia de dolor 0 alta sensibilidad en el muñón, tener diagnóstico médico actual o pasado que revele una afectación en el equilibrio, como un trastorno vestibular o neurológico, tomar 
medicamentos que afecten el equilibrio y síntomas de mareo o vértigo. El promedio de edad en este grupo fue 32,35 años (SD: 3,2, rango 29-46 años), en masa corporal el promedio fue de $78,25 \mathrm{~kg}$ (SD: 6,5 , rango $66-89 \mathrm{~kg}$ ), talla promedio $176 \mathrm{~cm}$ (SD: 2,7, rango 160-180 cm), Fig. 1.

\subsection{Protocolo}

Los participantes se ubicaron sobre las plantillas del sistema Pedar ${ }^{\circledR}$ (Novel, Alemania), el cual permite obtener información de la fuerza que interactúa entre el pie y la suela del zapato, así como la medición de la distribución de la fuerza de contacto y la ubicación del COP.

El sistema consta de 198 sensores capacitivos (99 en cada plantilla), software y sistema de visualización. Las plantillas de presión se colocaron sobre el piso para medir el COP; de esta forma se controló la posición de los pies y también la bipedestación durante la toma de muestras, utilizando la primera condición del protocolo del test de Romberg.

La ubicación de las plantillas hace que los pies del sujeto tengan una distancia de separación de $150 \mathrm{~mm}$ y un ángulo de progresión de $8^{\circ}$ [15]. Las personas que participaron en el estudio utilizaron la ropa y los zapatos que normalmente usan, los amputados emplearon su prótesis regular, a la que no se le hizo ninguna alteración mecánica. Para el momento de la medición, el paciente puso cada pie sobre cada una de las plantillas, con la mirada al frente a una pared blanca situada a 7,5 m, con los brazos a los lados, los ojos abiertos, y se le dio la instrucción de permanecer lo más quieto posible o estático (posición de medición) durante 15 segundos. A cada sujeto se le tomaron tres veces los datos, con un intervalo de medición de 10 minutos, Fig. 2.

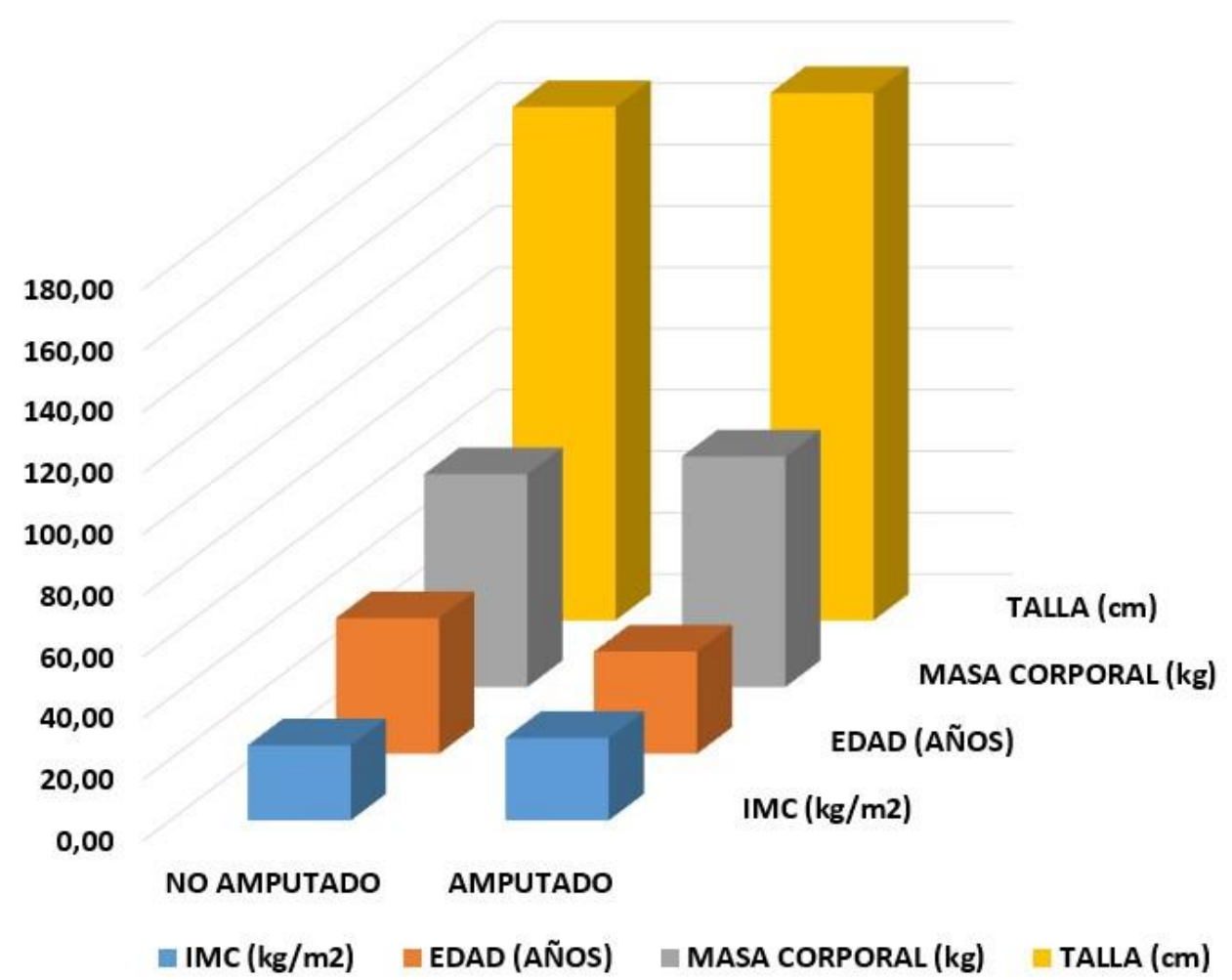

Fig. 1. Distribución de los grupos estudiados: no amputado (grupo control) y amputado. Los parámetros de IMC, edad, masa corporal, talla, son semejantes entre los grupos. Fuente: elaboración propia. 


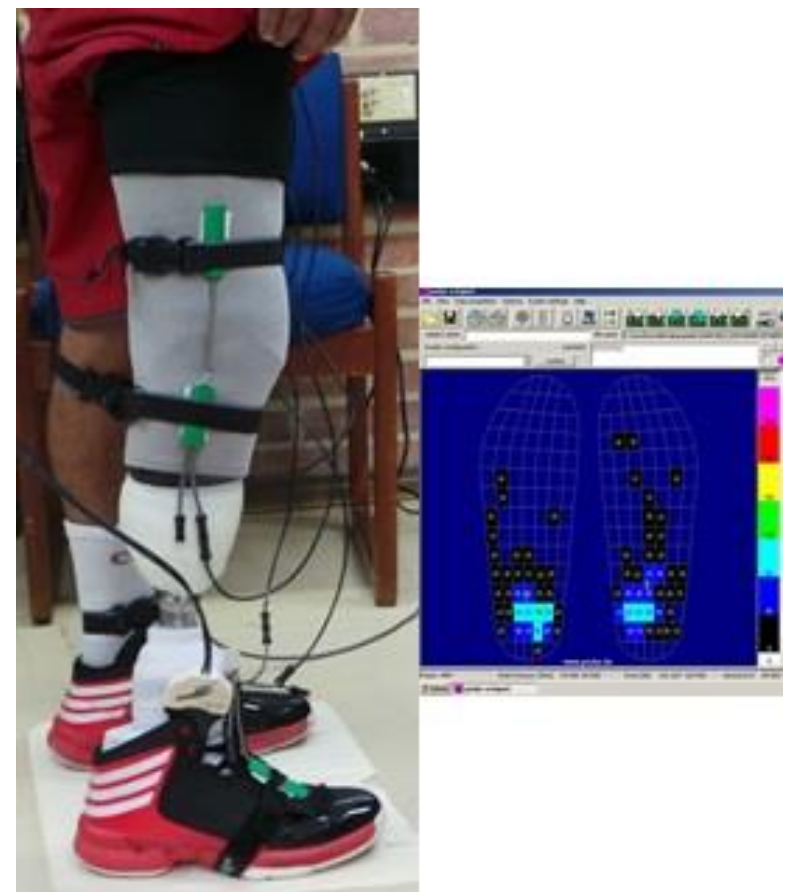

Fig. 2. Medición de COP en un sujeto amputado, que se ubica sobre las plantillas de medición Fuente: elaboración propia.

\subsection{Densidad Espectral de Potencia (DEP)}

Se registró la presión plantar y la ubicación del COP con una frecuencia de muestreo de $50 \mathrm{~Hz}$. Con el fin de reducir las señales indeseadas producto del comportamiento inconsistente de los sujetos al comienzo de la prueba, se eliminaron los primeros cinco (5) segundos de cada grabación. Las señales se filtraron con un filtro de paso bajo de Butterworth de $4^{\circ}$ orden de retraso cero con una frecuencia de corte de $10 \mathrm{~Hz}$. Lo anterior se desarrolló teniendo en cuenta la propuesta de Winter al analizar el patrón de bipedestación, reforzada por Wachowiak, quienes sugieren que la señal a analizar se encuentra en frecuencias inferiores a la frecuencia de corte del filtro [16], [17]. La pierna amputada se tomó como el lado derecho.

Para el cálculo de la Densidad Espectral de Potencia (DEP) se utilizaron los 454 datos de cada sujeto, que corresponden a 9,08 segundos de la señal COP en las direcciones antero-posterior y medial-lateral; de esta forma, se determinó la distribución de la potencia de las señales a lo largo de un intervalo de frecuencias. La DEP fue calculada con el periodograma modificado de Welch. Esta función estima la DEP al promediar varios periodogramas obtenidos de la misma señal en ventanas temporales consecutivas con un cierto porcentaje de solapamiento, lo que suaviza el espectro de una señal en la medida en que permite disminuir la varianza entre estimaciones y viene dada por la expresión (1):

$$
x i(n)=x(n+i D) n=0,1, \ldots N-1
$$

Se utilizó la ventana de Hamming con un solapamiento del $25 \%$, estas son ventanas de 64 muestras y de aproximadamente 1,28 segundos. Como es el propuesto por Welch, el número de secciones $\mathrm{K}$ de longitud $\mathrm{L}$ es $\mathrm{K}=2 \mathrm{~N} / \mathrm{L}-1$.

El espectro de la señal de la PSD estimada abarca el intervalo [0, Fs / 2], donde Fs es la frecuencia de muestreo de 50 $\mathrm{Hz}$. Dado que la componente frecuencial está contenida por debajo de los $3 \mathrm{~Hz}$, se establecieron las gráficas en el intervalo de frecuencia de [02,5] Hz, [5]. 


\subsection{Frecuencia promedio normalizada}

La frecuencia promedio angular normalizada fue estimada a partir del espectro de potencia de la señal del COP en el dominio del tiempo, usando una ventana rectangular y con la frecuencia de muestreo dada en Hz. La potencia promedio dentro del rango especificado se obtuvo al calcular la potencia de cada compartimento a partir de la multiplicación del ancho de frecuencia de cada compartimiento y la DEP; luego se buscaron todos los elementos dentro del rango de frecuencia definido; para finalizar, se calculó la potencia total en el momento central dentro de este, que se refiere a la frecuencia promedio.

\subsection{Frecuencia mediana normalizada}

La frecuencia mediana se define como la frecuencia a la cual el espectro de potencia se divide en dos áreas iguales mediante una aproximación integral rectangular.

El cálculo de la potencia mediana se realiza obteniendo inicialmente la potencia de la DEP, luego se aplica una integración rectangular acumulativa, se establecen los bordes medios entre cada estimación de frecuencia, se busca la potencia integrada para el rango de frecuencia definido $y$, finalmente, se calcula la potencia al restar la parte baja de la parte alta entre el rango de frecuencia hallado anteriormente. La frecuencia mediana es aquella que divide la potencia por igual.

\subsection{Ancho de banda del $99 \%$ de frecuencia normalizada}

El ancho de banda ocupado del $99 \%$ de la frecuencia angular normalizada se obtiene a partir del espectro de potencia de la señal del COP en el dominio del tiempo, empleando una ventana rectangular $\mathrm{y}$ considerando la frecuencia de muestreo.
Para calcular el ancho de banda ocupado, primero se debe evaluar la estimación de densidad espectral de potencia utilizando el método del periodograma con una ventana Kaiser; luego de esto, la DEP se integra con una aproximación rectangular. El ancho de banda se calcula a partir de las interceptaciones de frecuencia, donde la potencia integrada cruza el $0,5 \%$ y el $99,5 \%$ de la potencia total en el espectro.

\subsection{Ancho de banda de $3 \mathrm{~dB}$ de frecuencia normalizada}

El ancho de banda de la potencia media (banda de $3 \mathrm{~dB}$ ) de la frecuencia angular normalizada se consigue del espectro de potencia de la señal del COP en el dominio del tiempo, usando una ventana rectangular y a la frecuencia de muestreo.

Para calcular el ancho de banda de $3 \mathrm{~dB}$, primero se estima un espectro de potencia utilizando el método del periodograma con una ventana de Kaiser. A continuación, se calcula un nivel de referencia como el nivel máximo de potencia del espectro de potencia o "frecuencia pico". El ancho de banda se calcula a partir de las intercepciones de frecuencia donde el espectro cae por debajo del nivel de referencia en $3 \mathrm{~dB}$, o se encuentra hacia el final del espectro (es decir el que esté más cerca).

\section{RESULTADOS}

El desplazamiento del COP en función del tiempo en los grupos estudiados se observa en la Fig. 3. Ya que el análisis es no-lineal, no se requiere observar la distribución estadística de los datos. La relevancia del estudio empleando Densidad Espectral de Potencia (DEP) radica en el análisis frecuencial. 
EJE MEDIAL-LATERAL

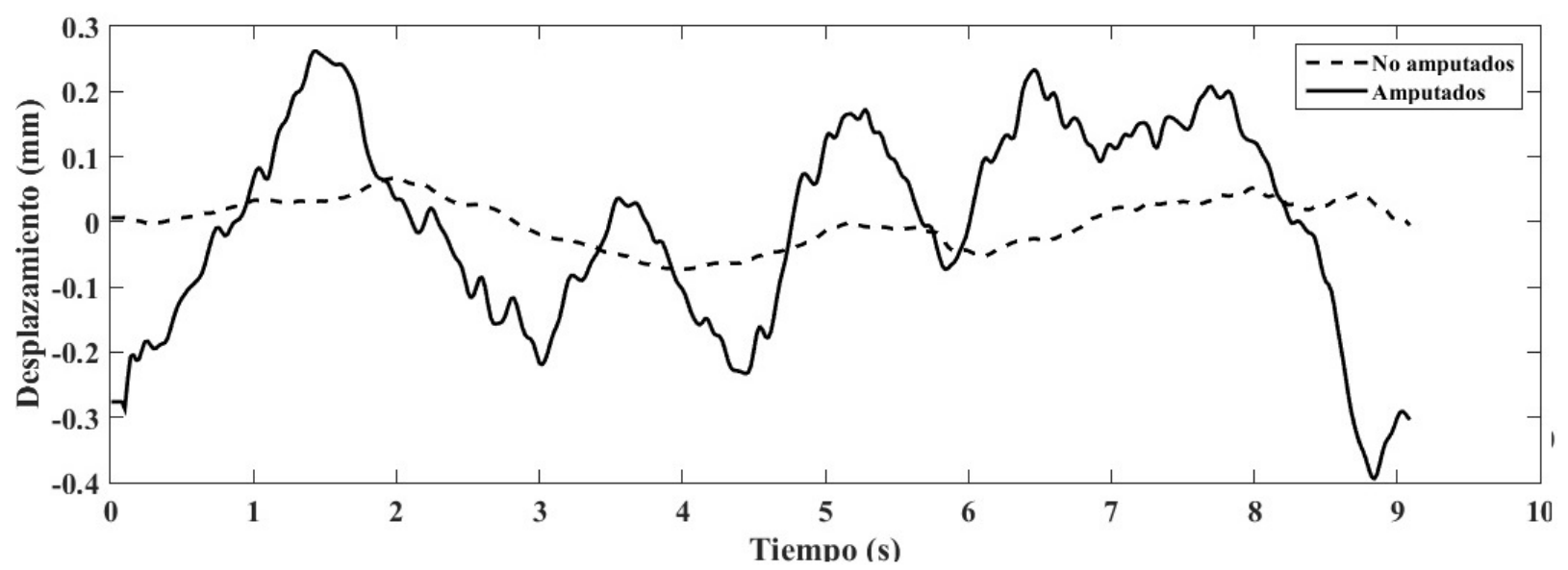

EJE ANTERO-POSTERIOR

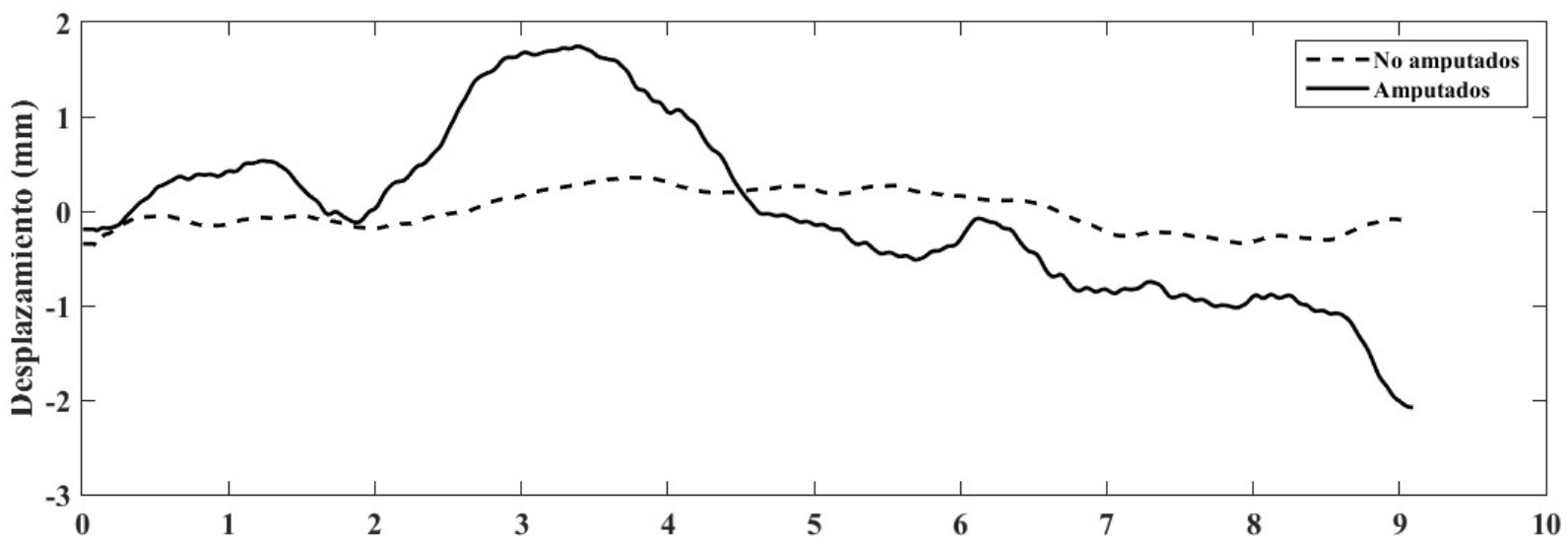

Tiempo (s)

Fig. 3. Desplazamiento del COP en función del tiempo presentado por los dos grupos medidos, se muestra en los dos ejes, medial-lateral y antero-posterior. Fuente: elaboración propia.

En el grupo amputados la frecuencia se estableció entre 0 y $1,523 \mathrm{~Hz}$ tanto para la pierna izquierda como para la pierna derecha en el desplazamiento mediallateral, en el desplazamiento anteroposterior la pierna izquierda está entre $0 \mathrm{y}$ $4,319 \mathrm{~Hz}$, y para el desplazamiento antero- posterior la pierna derecha entre 0 y $2,813 \mathrm{~Hz}$. Igualmente, la DEP del grupo de amputados mantuvo significativamente mayor potencia en ambas piernas y desplazamientos con respecto al grupo de no amputados, Fig. 4. 

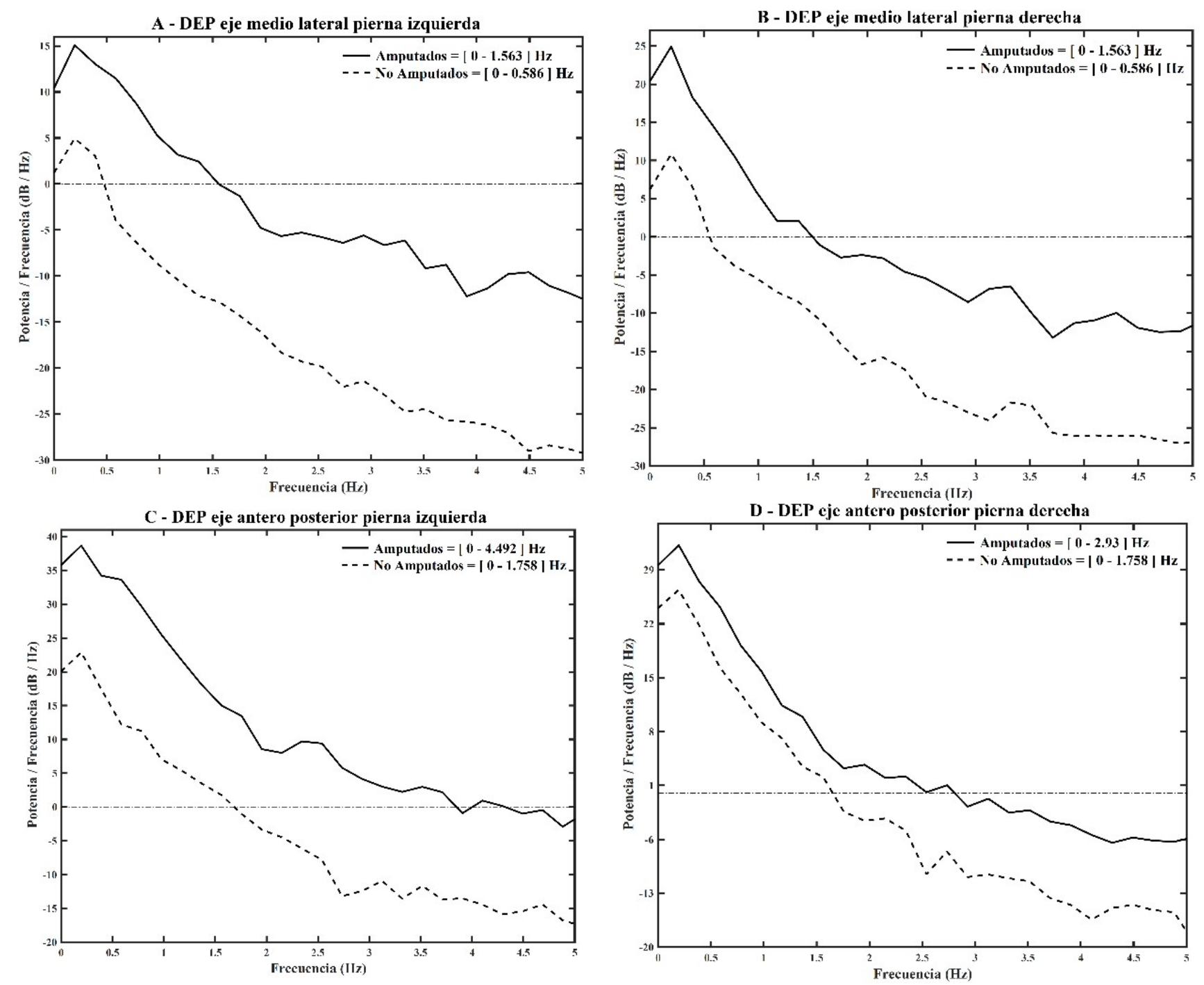

Fig. 4. DEP de los grupos amputados y no amputados y de cada pierna Fuente: elaboración propia.

En el grupo de los amputados la frecuencia promedio se estableció en 0,738 $\mathrm{Hz}$, con una potencia de $0,043 \mathrm{~dB}$ en el desplazamiento medial-lateral de la pierna izquierda; en el desplazamiento mediallateral de la pierna derecha, la frecuencia promedio se estableció en $0,406 \mathrm{~Hz}$, con una potencia de 0,772 dB; en el desplazamiento antero-posterior de la pierna izquierda la frecuencia es de $0,385 \mathrm{~Hz}$, con una potencia de $12,28 \mathrm{~dB}$, mientras que en la derecha estuvo en $0,419 \mathrm{~Hz}$, con una potencia de 1,404 dB. El grupo de amputados mantuvo significativamente mayor potencia en ambas piernas y desplazamientos con respecto al grupo de no amputados, Fig. 5. 

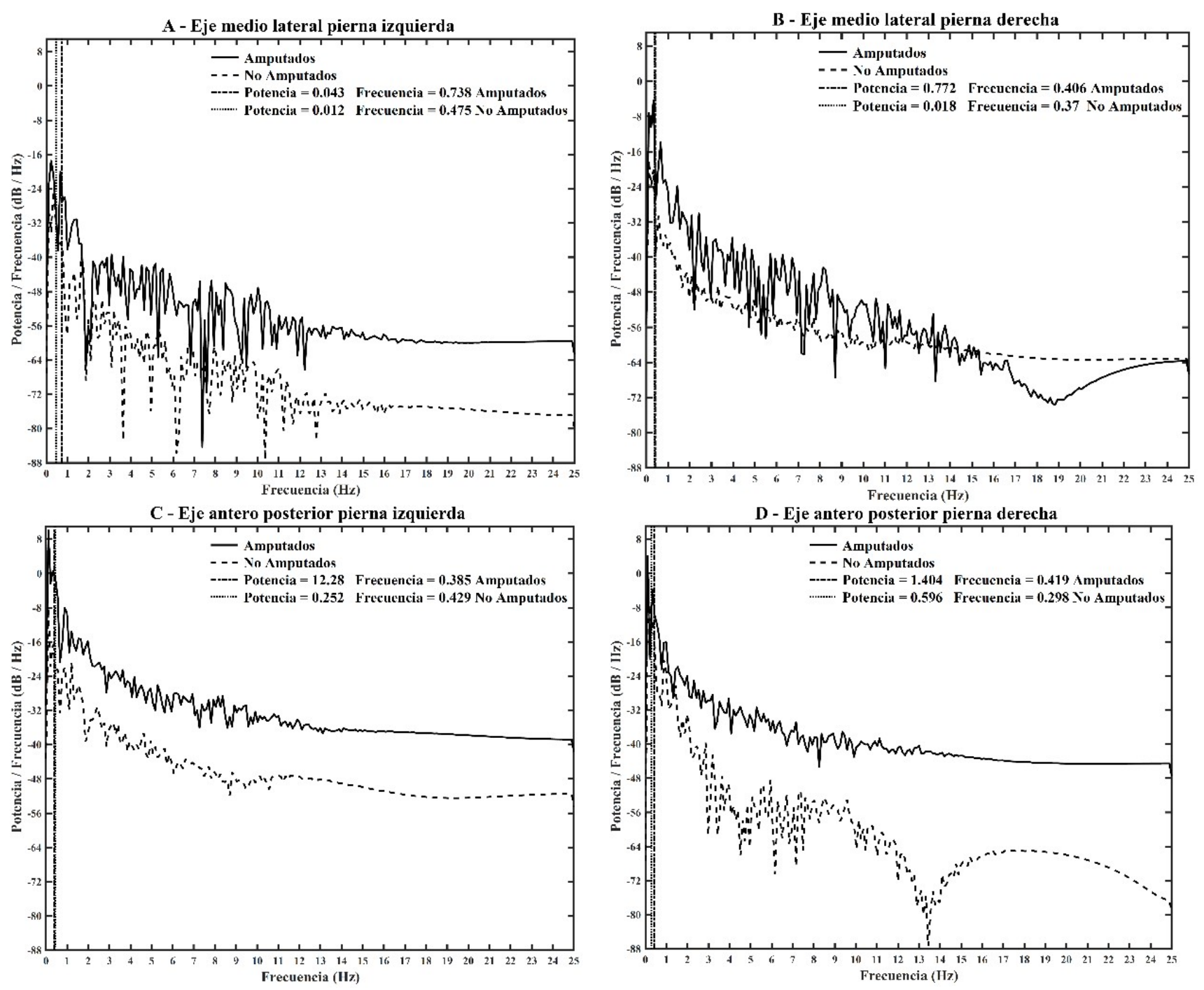

Fig. 5. Frecuencia promedio del COP en cada grupo y cada pierna

Fuente: elaboración propia.

La frecuencia mediana se estableció en $0,359 \mathrm{~Hz}$ para el grupo de amputados, con una potencia de $0,043 \mathrm{~dB}$ en el desplazamiento medial-lateral de la pierna izquierda; para la pierna derecha en el mismo eje, la frecuencia mediana se estableció en $0,185 \mathrm{~Hz}$, con una potencia de 0,772 dB. El desplazamiento anteroposterior de la pierna izquierda con una potencia de 12,28 dB sostuvo una frecuencia de $0,265 \mathrm{~Hz}$; en el caso de la pierna derecha en este eje, la potencia fue de $1,404 \mathrm{~dB}$ a una frecuencia de $0,253 \mathrm{~Hz}$.

En el eje medio lateral, el grupo de amputados mantuvo significativamente mayor potencia en ambas piernas y desplazamientos con respecto al grupo de no amputados, Fig. 6. 

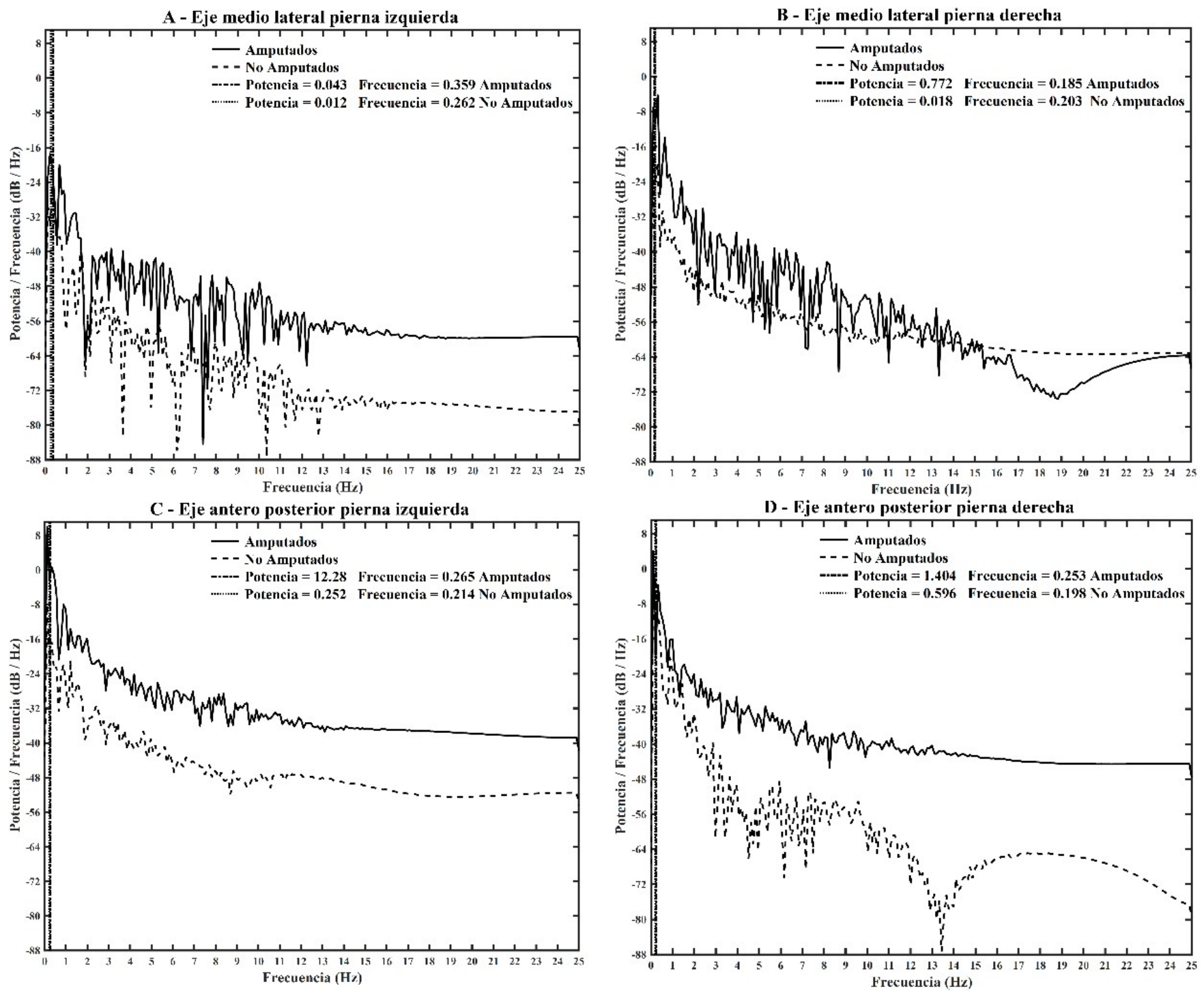

Fig. 6. Frecuencia mediana del cop en cada grupo y cada pierna Fuente: elaboración propia.

En los amputados, el $99 \%$ de la frecuencia se encontró entre 0 y $8,603 \mathrm{~Hz}$, con una potencia de $0,0589 \mathrm{~dB}$; en el desplazamiento medial-lateral de la pierna izquierda y derecha se estableció entre 0 y $6,251 \mathrm{~Hz}$, con una potencia de 0,0565 dB.

En el desplazamiento antero-posterior, la pierna izquierda se sitúo entre $0 \quad \mathrm{y}$
$3,752 \mathrm{~Hz}$, con una potencia de $0,0571 \mathrm{~dB}$, la pierna derecha estuvo entre 0 y $6,202 \mathrm{~Hz}$, con una potencia de 0,0567 dB. En ambas piernas y desplazamientos, el grupo de amputados mantuvo una potencia no muy diferente de la del grupo de no amputados, Fig. 7. 

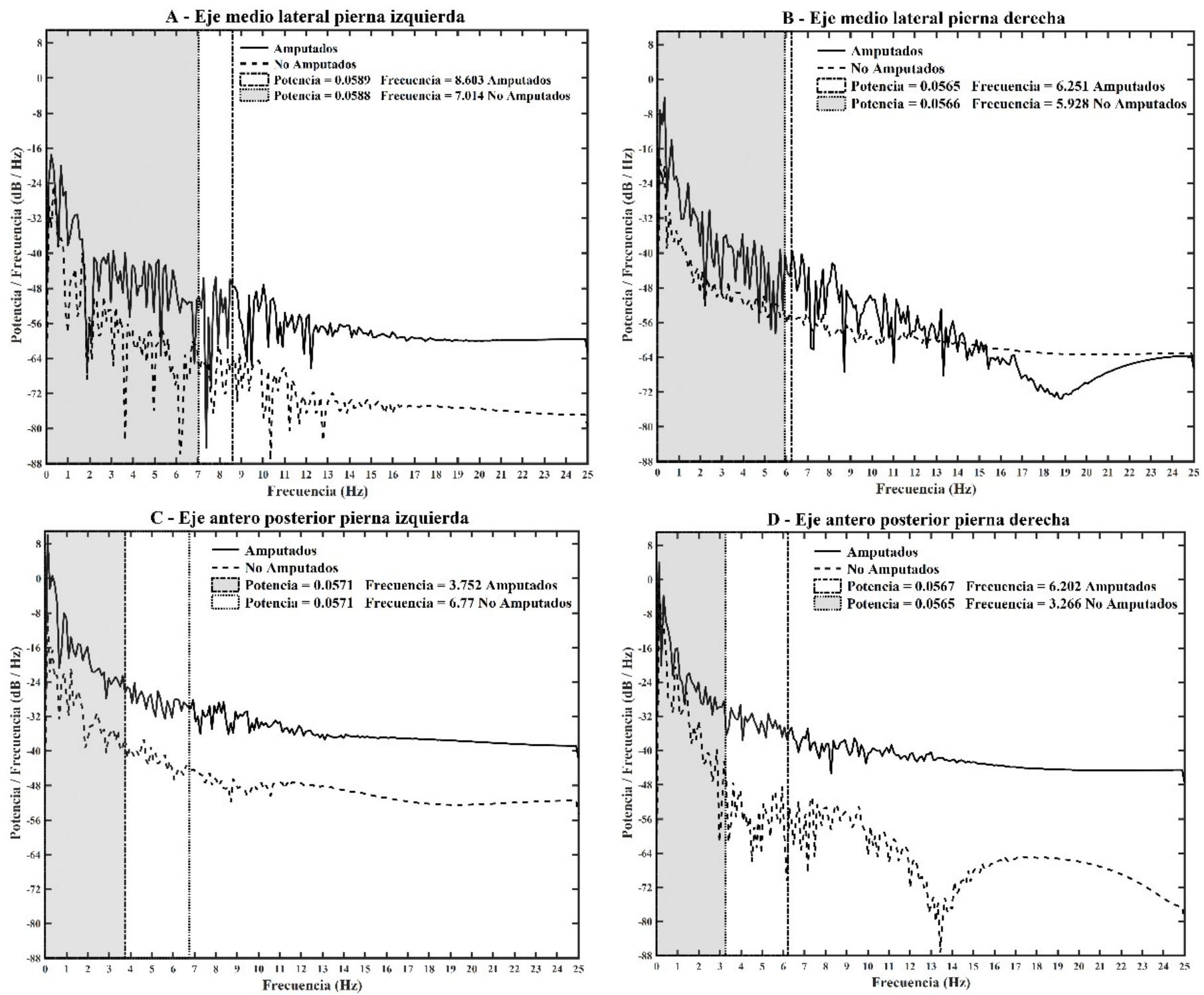

Fig. 7. Ancho de banda del $99 \%$ de frecuencia del cop en cada grupo y cada pierna

Fuente: elaboración propia.

La frecuencia pico en el grupo de amputados se estableció entre 0 y $0,109 \mathrm{~Hz}$, con una potencia de $0,1604 \mathrm{~dB}$ en el desplazamiento medial-lateral para la pierna izquierda, y para derecha la frecuencia pico estuvo entre 0 y $0,087 \mathrm{~Hz}$, con una potencia de $0,123 \mathrm{~dB}$. En el desplazamiento antero-posterior de la pierna izquierda, la frecuencia pico se sitúo entre 0 y $0,118 \mathrm{~Hz}$, con una potencia de $0,1276 \mathrm{~dB}$; la pierna derecha estuvo entre 0 y $0,099 \mathrm{~Hz}$, con una potencia de 0,1912 dB.
El grupo de amputados mantuvo ligeramente mayor potencia con respecto al grupo de no amputados, esto solamente en el desplazamiento antero-posterior de ambas piernas, Fig. 8 .

De los resultados anteriores, se observa claramente que existen diferencias entre la distribución de las frecuencias en los grupos de estudio, con una mayor oscilación hacia el eje anteroposterior de la pierna izquierda con respecto a la derecha del grupo amputado, producto de la compensación que debe realizar la pierna no amputada para 
mantener la posición bípeda estática. Una mayor compensación o dominio de la postura erguida es un requisito fundamental para la realización de las tareas de la vida diaria, como abrir puertas, recoger objetos, trasladarse, etc. Por lo tanto, esa descompensación resulta del uso de la prótesis hace que la distribución de frecuencia-potencia muestre mayores valores; en el caso de la DEP, siempre fue mayor en el grupo de amputados. Esto hace que los cambios que se observaron en las figuras anteriores en la distribución de frecuencia y potencia confirmen la no estacionariedad del COP, cuando se analiza durante intervalos prolongados de tiempo.

La mayor parte de la potencia promedio del COP está contenida en el plano anteroposterior.
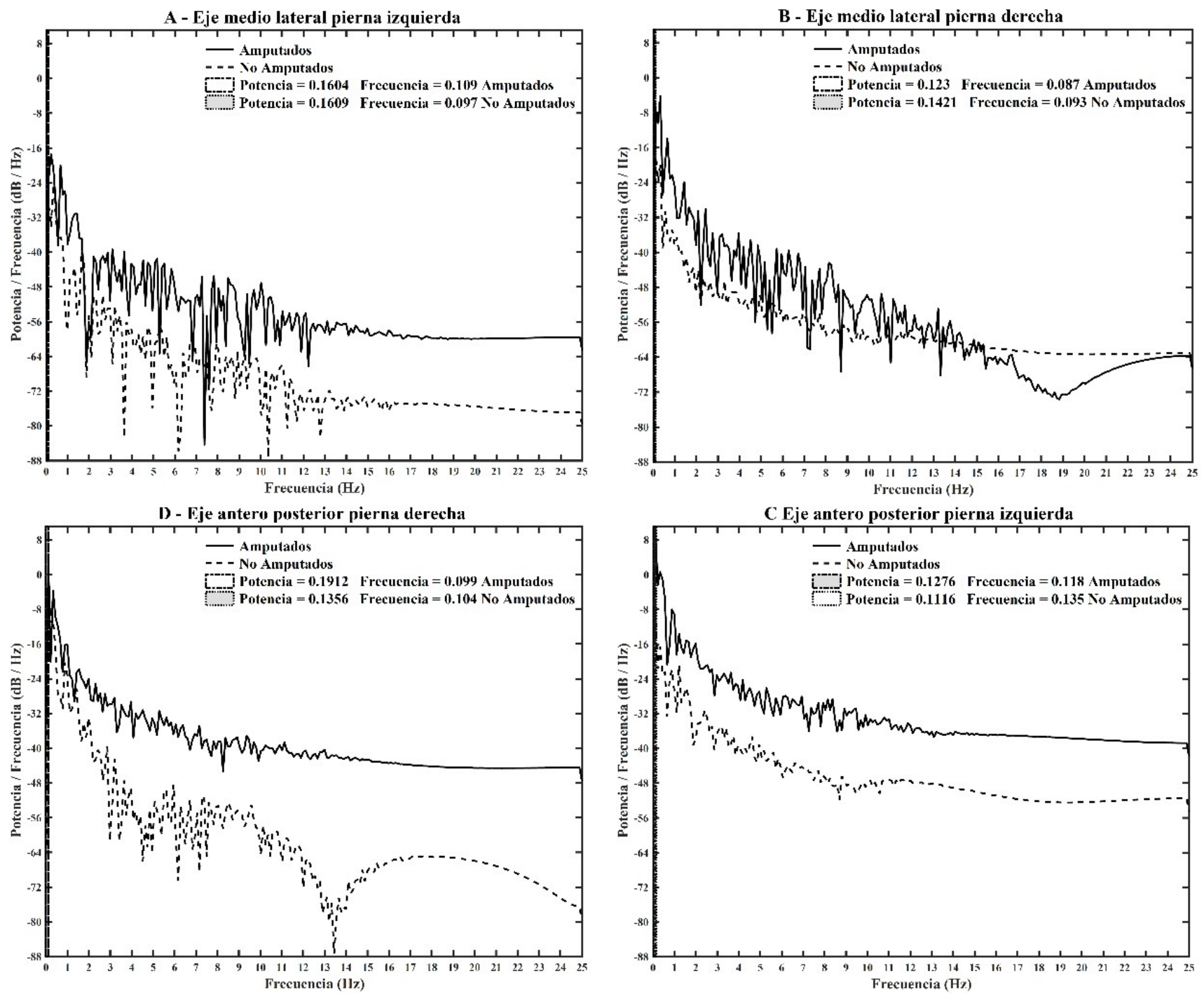

Fig. 8. Ancho de banda de $3 \mathrm{~dB}$ de frecuencia del COP en cada grupo y cada pierna Fuente: elaboración propia. 


\section{DISCUSIÓN}

Se obtuvo la densidad espectral de potencia (DEP) para dos grupos estudiados: amputados y no amputados.

En posición de bipedestación estática en el desplazamiento mediolateral, los amputados muestran un rango de frecuencia entre 0 y $1,523 \mathrm{~Hz}$ en ambas extremidades, es decir, exhiben baja oscilación en este eje, esto corrobora lo resaltado por [16]. En cuanto a la dirección anteroposterior, la densidad espectral es mayor en la pierna izquierda (no amputada), lo que indica mayor oscilación en este lado; los datos obtenidos del análisis se encuentran afines con los reportados en estudios realizados por [19]-[20].

La potencia promedio en los amputados en medio-lateral es menor que en anteroposterior; una posible causa de esto es la carencia de tobillo anatómico y todas sus estructuras en el lado amputado, lo que produce que el mecanismo de compensación de descarga de peso se realice con la articulación de cadera, situación analizada por [21]. En los no amputados se presenta menor potencia que en los amputados en todas las condiciones de prueba.

Los hallazgos sugieren que los mecanismos de estabilización a toda costa intentan mantener el CG igual al COM.

El uso de estrategias para mantener el equilibrio y minimizar las influencias desestabilizantes impide que el COP sobresalga de los límites de estabilidad.

Según la intensidad del estímulo desestabilizador, se cuenta con diferentes tipos de estrategias: la estrategia de tobillo, la estrategia de cadera, la estrategia de paso, la estrategia de suspensión y la estrategia de cambio de peso. Los resultados opuestos entre los dos grupos sugieren la existencia de una diferencia en el uso de estrategias de control de equilibrio. Debido a la carencia del tobillo anatómico y por tanto de todas las estructuras sensorimotoras que involucran este segmento anatómico, los amputados transtibiales son incapaces de mantener la estabilidad postural con una estrategia de tobillo en el lado protésico. De allí que, para mantener la estabilidad postural, se usan las estructuras restantes; de esta forma, utilizan una estrategia postural modificada que corresponde al uso de la rodilla.

Para controlar la estabilidad y el equilibrio de esa articulación, el amputado utiliza la musculatura del glúteo y de la parte posterior del muslo [5]. Entre tanto, las personas no amputadas suelen utilizar la estrategia de tobillo para las superficies estables.

\section{CONCLUSIONES}

El análisis frecuencial es una técnica útil para cuantificar la variabilidad general del desplazamiento del COP, así como los componentes específicos asociados con efectos patológicos o fisiológicos que causan alteraciones en la estabilidad corporal.

Varias investigaciones han hecho un análisis espectral de potencia del COP. Para el cálculo de la estimación espectral, diversos estudios han utilizado la Transformada de Fourier, pero esta herramienta supone que la señal es estacionaria y su funcionamiento no es el mejor en señales no estacionarias, como es el caso de la señal COP. De hecho, el algoritmo de Fourier no tiene en cuenta el sesgo de estimación y la varianza, por lo que su aplicación conduce a una estimación del espectro del COP que no converge [22][24]. Una forma correcta de estimar la densidad espectral de potencia (DEP) de una señal se relaciona con un estimador imparcial y consistente [25], [26]. Por lo anteriormente mencionado, en esta investigación se hizo un análisis lineal que calcula la DEP de la señal COP con el método de Welch.

La gráfica de distribución de frecuenciapotencia muestra picos que se extienden en ambas dimensiones de estas, que capturan la dinámica del control postural. Los 
cambios en la distribución de frecuencia y potencia confirman la no estacionariedad del COP cuando se analiza durante intervalos de tiempo más largos. La mayor parte de la potencia promedio del COP está contenida en el plano antero-posterior.

La disminución de la potencia promedio contenida en el plano medial-lateral probablemente es producto de la rigidez anatómica de las articulaciones del tobillo y subastragalina en la dirección mediallateral y la pequeña contribución de los abductores y aductores de cadera durante la carga de cadera: mecanismo de descarga.

A pesar de la mayor variación en los picos de potencia en el tiempo, se observó un patrón consistente en la potencia media y mediana con respecto a la frecuencia media y mediana y el ancho de banda espectral. El contenido de frecuencia del COP disminuye y se desplaza hacia frecuencias más bajas a medida que aumenta la potencia del COP, así se establece una relación inversa entre la frecuencia media y mediana con la potencia media y mediana respectivamente.

$\mathrm{El}$ factor de proporcionalidad inversa en la pierna no amputada, al ser evaluado en el plano antero-posterior, es menor al del plano medial-lateral. Esta discrepancia puede resaltar la importancia de la información propioceptiva en el control de estabilidad postural.

Debido a la disminución de información propioceptiva, como consecuencia de la amputación, las estrategias de control de equilibro usadas por los amputados difieren de las empleadas por los no amputados.

En un futuro se espera ampliar el campo de estudio en el análisis de estabilidad dinámica, incluyendo sujetos con diversas causas de amputación y diferentes tipos de prótesis, para así abarcar un grupo poblacional con características más variadas.

\section{AGRADECIMIENTOS}

Los autores expresan sus agradecimientos al Servicio de Amputados del Hospital Militar Central y a las personas que participaron en la investigación.

\section{REFERENCIAS}

[1] L. H. Lugo Agudelo y V. Seijas, "La discapacidad en Colombia: una mirada global," Rev. Colomb. Med. Física y Rehabil., vol. 22, no. 2, pp. 164-179, 2012. Disponible en: URL

[2] Dirección Contra Minas, "Víctimas de minas antipersonal y municiones sin explosionar", Presidencia de la República de Colombia, 2018. Disponible en: URL

[3] L. A. Luengas Contreras, E. Camargo Casallas, y D. Guardiola, "Modelado y simulación de la marcha protésica usando modelo en 3D de una prótesis transtibal," Rev. Ciencias la Salud, vol. 16, no. 1, p. 82100, Jan. 2018.

https://doi.org/10.12804/revistas.urosario.edu. co/revsalud/a.6492

[4] J. Ospina y F. Serrano, "El paciente amputado: Complicaciones en su proceso de rehabilitación”, Rev. Ciencias la Salud, vol. 7, no 2, pp. 36-46, May 2009. Disponible en: $\underline{\mathrm{URL}}$

[5] L. A. Luengas C. y D. C. Toloza, Análisis de estabilidad en amputados transtibiales unilaterales. Bogotá: UD Editorial, 2019. Disonible en: URL

[6] R. M. Palmieri, C. D. Ingersoll, M. B. Stone, y B. A. Krause, "Center-of-Pressure Parameters Used in the Assessment of Postural Control," J. Sport Rehabil., vol. 11, no. 1, pp. 51-66, Feb. 2002. https://doi.org/10.1123/jsr.11.1.51

[7] J. A. Raymakers, M. M. Samson, y H. J. J. Verhaar, "The assessment of body sway and the choice of the stability parameter(s)," Gait Posture, vol. 21, no. 1, pp. 48-58, Jan. 2005. https://doi.org/10.1016/j.gaitpost.2003.11.006

[8] P. J. Loughlin, M. S. Redfern y J. M. Furman, "Nonstationarities of Postural Sway", IEEE Eng. Med. Biol. Mag., vol. 22, no. 2, pp. 69-75, Apr. 2003. https://doi.org/10.1109/MEMB.2003.1195699

[9] H. Van Der Kooij, E. Van Asseldonk, y F. C. T. Van Der Helm, "Comparison of different methods to identify and quantify balance control", J. Neurosci. Methods, vol. 145, no. 12, pp. 175-203, Jun. 2005. 
https://doi.org/10.1016/j.jneumeth.2005.01.00 $\underline{3}$

[10] C. Fujimoto, N. Egami, S. Demura, T. Yamasoba y S. Iwasaki, "The effect of aging on the center-of-pressure power spectrum in foam posturography", Neurosci. Lett., vol. 585, pp. 92-97, Jan. 2015.

https://doi.org/10.1016/j.neulet.2014.11.033

[11] H. Qiu y S. Xiong, "Center-of-pressure based postural sway measures: Reliability and ability to distinguish between age, fear of falling and fall history", Int. J. Ind. Ergon., vol. 47, pp. 37-44, May. 2015. https://doi.org/10.1016/j.ergon.2015.02.004

[12] A. J. Orozco-Naranjo y P. A. MuñozGutiérrez, "Detección de latidos cardiacos patológicos y normales utilizando transformada por paquetes wavelet, máquinas de soporte vectorial y perceptrón multicapa”, TecnoLógicas, no. 31, pp. 73-91,

Nov. 2011.

https://doi.org/10.22430/22565337.102

[13] U. Oppenheim, R. Kohen-Raz, D. Alex, A. Kohen-Raz, y M. Azarya, "Postural characteristics of diabetic neuropathy", Diabetes Care, vol. 22, no. 2, pp. 328-332, Feb. 1999.

http://dx.doi.org/10.2337/diacare.22.2.328

[14] P. R. Cavanagh, G. G. Simoneau, y J. S. Ulbrecht, "Ulceration, unsteadiness, and uncertainty: the biomechanical consequences of diabetes mellitus", J. Biomech., vol. 26, no. 1 , pp. 23-40, 1993. http://dx.doi.org/10.1016/0021-9290(93)90077$\underline{\mathrm{R}}$

[15] M. Lord y D. M. Smith, "Foot loading in amputee stance", Prosthet. Orthot. Int., vol. 8, no. 3, pp. 159-64, Dic. 1984. Disponible en: URL

[16] D. A. Winter, Biomechanics and motor control of human movement, 4th ed. New Jersey: John Wiley \& sons, Inc, 2009. http://dx.doi.org/10.1002/9780470549148

[17] D. C. Hay y M. P. Wachowiak, "Analysis of free moment and center of pressure frequency components during quiet standing using magnitude squared coherence", Hum. Mov. Sci., vol. 54, pp. 101-109, Aug. 2017. https://doi.org/10.1016/j.humov.2017.04.002

[18] H. Nadollek, S. Brauer, y R. Isles, "Outcomes after trans-tibial amputation: the relationship between quiet stance ability, strength of hip abductor muscles and gait", Physiother. Res. Int., vol. 7, n. ${ }^{\circ}$, pp. 203-214, Nov. 2002.

http://dx.doi.org/10.1002/pri.260

[19] P. R. Rougier y J. Bergeau, "Biomechanical Analysis of Postural Control of Persons with
Transtibial or Transfemoral Amputation", Am. J. Phys. Med. Rehabil., vol. 88, no. 11, pp. 896-903, Nov. 2009. http://dx.doi.org/10.1097/PHM.0b013e3181b3 $\underline{31 a f}$

[20] P. Hlavackova, C. Franco, B. Diot, y N. Vuillerme, "Contribution of Each Leg to the Control of Unperturbed Bipedal Stance in Lower Limb Amputees: New Insights Using Entropy”, PLoS One, vol. 6, no. 5, May. 2011. https://doi.org/10.1371/journal.pone.0019661

[21] D. A. Winter, F. Prince, J. S. Frank, C. Powell, y K. F. Zabjek, "Unified theory regarding $\mathrm{A} / \mathrm{P}$ and $\mathrm{M} / \mathrm{L}$ balance in quiet stance.", J. Neurophysiol., vol. 75, n. ${ }^{\circ} 6$, pp. 2334-43, Jun. 1996.

https://doi.org/10.1152/jn.1996.75.6.2334

[22] R. Shiavi, Introduction to applied statistical signal analysis: guide to biomedical and electrical engineering applications, 3rd ed. Burlington: Academic Press, 2007. Disponible en: URL

[23] J. S. Bendat y A. G. Piersol, Random data: analysis and measurement procedures. Hoboken, NJ, USA: John Wiley \& Sons, Inc., 2010. http://dx.doi.org/10.1115/1.3269865

[24] J. P. Carroll y W. Freedman, "Nonstationary properties of postural sway", J. Biomech., vol. 26, no. 4-5, pp. 409-16, Apr. 1993.

https://doi.org/10.1016/0021-9290(93)90004-x

[25] M. G. Carpenter, J. S. Frank, D. A. Winter, y G. W. Peysar, "Sampling duration effects on centre of pressure summary measures.", Gait Posture, vol. 13, no. 1, pp. 35-40, Feb. 2001. https://doi.org/10.1016/s0966-6362(00)00093-x

[26] A. Nardone, M. Grasso, y M. Schieppati, "Balance control in peripheral neuropathy: Are patients equally unstable under static and dynamic conditions?", Gait Posture, vol. 23, no. 3, pp. 364-373, Apr. 2006. https://doi.org/10.1016/j.gaitpost.2005.04.002

\section{CONTRIBUCIÓN DE LOS AUTORES}

(D) 1 Participó activamente en la conceptualización, diseño y desarrollo de la investigación, así como en la elaboración, redacción y revisión final del manuscrito.

(D) 2 Participó activamente en la conceptualización, diseño y desarrollo de la investigación, así como en la elaboración, redacción y revisión final del manuscrito. 